\title{
Disease Reports
}

\section{First Report of Fruit Black Spot of Diospyros kaki Caused by Colletotrichum acutatum in Korea}

\author{
Jin-Hyeuk Kwon ${ }^{1 *}$ and Jinwoo Kim ${ }^{2,3}$ \\ ${ }^{1}$ Gyeongsangnam-do Agricultural Research and Extension Services, Jinju 660-360, Korea \\ ${ }^{2}$ Department of Applied Biology, Gyeongsang National University, Jinju 660-701, Korea \\ ${ }^{3}$ Institute of Agriculture and Life Science, Gyeongsang National University, Jinju 660-701, Korea \\ (Received on September 12, 2010; Accepted on November 5, 2010)
}

Sweet persimmon (Diospyros kaki), a fruit tree with its geographic origin in China, is cultivated widely in Korea and Japan, the leading producers worldwide. Black spots were observed on the fruit of sweet persimmon collected in a farmer's orchard in Dongeup, Changwon, Korea, from July to October in 2008 and 2009. The disease occurred on the fruit, and not on the twigs or suckers. The symptom typically started with small black specks or spots like black sesame seeds on the surface of the fruit. Some adjacent spots coalesced, but these did not enlarge or become depressed like anthracnose of sweet persimmon. The corky surface of the lesions became somewhat rough (Fig. 1A). Since Colletotrichum gloeosporioides was first identified in Korea on decayed sweet persimmon, the typical symptoms of anthracnose of sweet persimmon caused by C. gloeosporioides are showed in Fig. $1 \mathrm{E}$ for comparison.

The pathogenic fungus was isolated from diseased fruit and a representative isolate was deposited with the Korean Agricultural Culture Collection (KACC 45234), National Academy of Agricultural Science, Rural Development Administration, Suwon, Korea. The fungus grown on potato dextrose agar(PDA) produced whitish mycelia initially, which became dark gray; later, salmoncolored conidial masses formed (Fig. 1C). The optimal temperature for mycelia growth was $25^{\circ} \mathrm{C}$. The conidia were fusiform in shape, occasionally constricted medially, unlike the conidia of $C$. gloeosporioides, and they measured 8-17 × 3-4 $\mu \mathrm{m}$ (Fig. 1D and $\mathrm{F})$. The appressoria were pale to dark brown in color, clavate or slightly irregular in shape, and measured 8-11 $\times 4-6 \mu \mathrm{m}$. Setae were absent. For pathogenicity tests, fresh persimmon fruits were artificially inoculated with a conidial suspension $\left(2 \times 10^{4}\right.$ conidia/ $\mathrm{ml})$ of the pathogen obtained from PDA cultures. After incubation, the same fungal fruiting symptoms were reproduced, and the fungus was re-isolated based on these symptoms (Fig. 1B). To identify the causal fungus, the rDNA internal transcribed spacer (ITS) sequence was obtained using the method of White et al. (1999). The resulting 584-bp sequence was deposited in GenBank (Accession No. HQ185296). Phylogenetic analysis was performed using MEGA4 with the neighbor-joining method and TajimaNei distance model. Comparison with ITS rDNA sequences

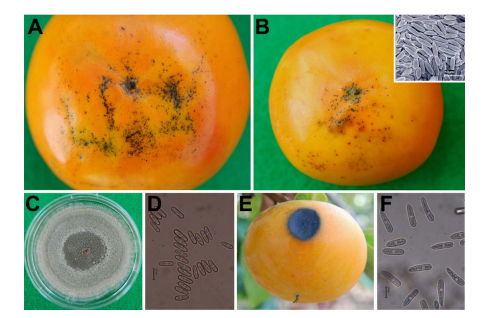

Fig. 1. Symptom of anthracnose of Diospyros kaki and morphological characteristics caused by Colletotrichum acutatum on matured fruits. A: Naturally infected fruits caused by $C$. acutatum. B: Symptoms after artificial inoculation. A small box shows scanning electron microscopic image of conidia of the C. acutatum. C: Mycelial colony of the $C$. acutatum grown on PDA for 10 days. D: Conidia of the C. acutatum grown on PDA. E: Naturally infected fruits caused by $C$. gloeosporioides. F: Conidia of the C. gloeosporioides on diseased fruit in the field. Bars $=10 \mu \mathrm{m}$. showed $100 \%$ similarity with sequences of C.acutatum (GenBank Accession No. AB219033) (Fig. 2). Based on the mycological characteristics, molecular data, and pathogenicity to the host plant determined in this experiment, the fungus was identified as $C$. acutatum J. H. Simmonds. The measurements and taxonomic characters matched those of C.acutatum described by Williamson and Sutton (2010). C. acutatum has been reported to cause fruit rot on persimmon fruit in New Zealand (Lardner et al., 1999). Anthracnose disease caused by C. acutatum has not been reported on sweet persimmon in Korea (The Korean Society of Plant Pathology, 2009). To our knowledge, this is the first report of $C$. acutatum on sweet persimmon in Korea. In Korea, the economic importance of the disease on sweet persimmon fruit is limited, although the pathogen could constitute a risk for apples and grapes.

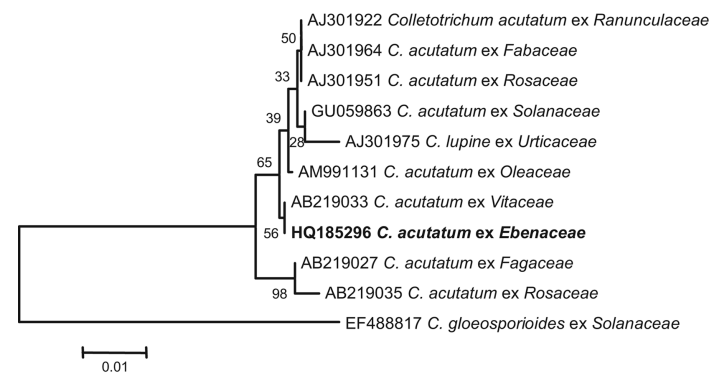

Fig. 2. Phylogenetic tree using ITS sequences showing closest known relatives of Colletotrichum acutatum including fruit black spot fungus infecting Diospyros kaki. DNA sequences from the NCBI nucleotide database were aligned using ClustalW. Numbers above the branches indicate the bootstrap values. Bars indicate number of nucleotide substitutions per site. The present isolate infecting sweet persimmon was marked in bold.

\section{Acknowledgment}

This work was carried out with the support of "Cooperative Research Program for Agriculture Science \& Technology Development (Project No. PJ005712)" Rural Development Administration, Korea.

\section{References}

Lardner, R., Johnston, P. R., Plummer, K. M. and Pearson, M. N. 1999. Morphological and molecular analysis of Colletotrichum acutatum sensu lato. Mycol. Res. 103:275-285.

The Korean Society of Plant Pathology. 2009. List of plant diseases in Korea, 5 th ed. 853 pp. (in Korean).

White, T. J., Bruns, T., Lee, S. and Taylor, J. W. 1990. Amplification and direct sequencing of fungal ribosomal RNA genes for phylogenetics. In: PCR Protocols: A guide to methods and applications, ed. by M. A. Innis, D. H. Gelfand, J. J. Sninsky, and T. J. White, pp. 315-322. Academic Press, Inc., New York.

Williamson, S. M. and Sutton, T. B. 2010. First report of anthracnose caused by Colletotrichum acutatum on persimmon fruit in the United States. Plant Dis. 94:634.

*Corresponding author (kwon825@korea.kr) 